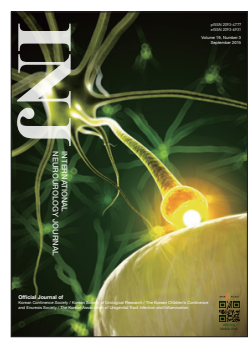

\title{
Underactive Bladder: Clinical Features, Urodynamic Parameters, and Treatment
}

\author{
Nathan Hoag, Johan Gani \\ Department of Urology, Austin Hospital, University of Melbourne, Melbourne, Australia
}

Purpose: Underactive bladder is a complex clinical condition that remains poorly understood due to limited literature. This study aimed to determine its prevalence among patients with voiding dysfunction, presenting symptoms, risk factors, urodynamic findings, and ongoing treatment.

Methods: A retrospective chart review of consecutive urodynamic studies performed on voiding dysfunction between 2012 and 2014 was conducted to identify patients with detrusor underactivity. Detrusor underactivity was defined by a bladder contractility index of less than 100. Charts and urodynamic tracings were examined for patient demographics, suspected risk factors, presenting symptoms, urodynamic parameters, and treatment undertaken. Descriptive statistics were utilized to analyze the data.

Results: The prevalence of detrusor underactivity in this study was $23 \%$ ( 79 of 343 ). Average age of the patients was 59.2 years (range, 19-90 years). Women represented 68.4\% (54 of 79) of the patients. The most common reported symptoms were urinary urgency $(63.3 \%)$, weak stream $(61.0 \%)$, straining $(57.0 \%)$, nocturia $(48.1 \%)$, and urinary frequency $(46.8 \%)$. Prior pelvic surgery and prior back surgery were noted in $40.5 \%$ and $19.0 \%$ of the patients, respectively. The most common management was intermittent self-catheterization in $54.4 \%$, followed by observation/conservative treatment in $25.3 \%$ and sacral neuromodulation in $12.7 \%$.

Conclusions: Although underactive bladder is a common condition, its precise diagnosis and treatment remain a challenge. Its symptoms significantly overlap with those of other bladder disorders, and hence, urodynamic evaluation is particularly useful in identifying patients with impaired detrusor contractility. This will help prevent mismanagement of patients with surgery or medical therapy, as that may worsen their condition. Much work needs to be done to better understand this condition and establish optimal management of patients.

Keywords: Lower Urinary Tract Symptoms; Urinary Retention; Urodynamics; Urinary Bladder

- Research Ethics: This study was approved by Institutional Ethics Review Board of Austin Health (LNR/15/Austin/194).

- Conflict of Interest: Johan Gani: Astellas (principal investigator), Medtronic (speaking honorarium). Except for that, no potential conflict of interest relevant to this article was reported.

\section{INTRODUCTION}

Underactive bladder (UAB) is a clinical entity often encountered by urologists, and is often associated with significant low- er urinary tract symptoms (LUTS) [1]. This condition is seen in both women and men, and can occur at any age. There exists a lack of consensus on the terminology, definition, and classification of UAB, which tends to create confusion and challenges in
Corresponding author: Nathan Hoag (iD http://orcid.org/0000-0002-6840-7152 Department of Urology, Austin Hospital, University of Melbourne, 49 Erin St, Richmond, Victoria, 3121 Australia

E-mail: nathanhoag2@gmail.com / Tel: +61-3-9428 2232 / Fax: +61-3-9428 2234 Submitted: July 14, 2015 / Accepted: August 28, 2015
This is an Open Access article distributed under the terms of the Creative Commons Attribution Non-Commercial License (http://creativecommons.org/licenses/by-nc/3.0/) which permits unrestricted non-commercial use, distribution, and reproduction in any medium, provided the original work is properly cited. 
its diagnosis and management. The International Continence Society (ICS) has defined the urodynamic finding of detrusor underactivity (DU) as "a contraction of reduced strength and/ or duration, resulting in prolonged bladder emptying and/or failure to achieve complete bladder emptying within a normal time span" [2]. However, the ICS has not yet defined UAB or UAB syndrome (UABS). A recent expert panel has proposed the following working definition for UAB: "The underactive bladder is a symptom complex suggestive of detrusor underactivity, and is usually characterized by prolonged urination time with or without a sensation of incomplete bladder emptying, usually with hesitancy, reduced sensation on filling, and a slow stream" [3].

The most commonly suggested symptoms of UAB include hesitancy, sensation of incomplete emptying, straining to void, as well as urgency, frequency, nocturia, incontinence (urge, stress, overflow), and recurrent urinary tract infections (UTI) [4]. Other cited symptoms are a slowed urinary stream, interrupted flow, and the need to rely on abdominal straining to void completely [1]. Importantly, an overlap of symptoms between overactive bladder $(\mathrm{OAB})$ and $\mathrm{UAB}$ has been noted, and accurate diagnosis is vital for appropriate treatment [4]. In a review of 1,179 patients undergoing urodynamics (UDS) for nonneurogenic LUTS, there was no statistically significant difference in symptoms among those with or without DU [5]. This typifies the potential challenges in characterizing UABS. It also highlights the utility of UDS in documenting detrusor function prior to initiating therapy, which is a potential pitfall in clinical practice, especially when UDS information is not readily obtainable.

The causes of UAB are varied, and may be neurogenic, myogenic, or underlying idiopathic etiologies. These include aging, bladder outlet obstruction (BOO), diabetes mellitus, neurologic diseases such as multiple sclerosis and Parkinson disease, spinal cord injury or pathology, infections affecting the nervous system, and iatrogenic causes, most often from prior pelvic or back surgery [6].

Treatment options for UAB are somewhat limited, especially when compared with the myriad of options available for OAB. Conservative measures, including timed/double voiding and straining to empty the bladder, may be appropriate for some patients. Urinary catheters are often employed in those cases where conservative therapy either fails or is not suitable [1]. Effective medical therapies for UAB have remained inadequate to date [7], while sacral neuromodulation (SNM) has shown some promise in the setting of nonobstructive urinary retention $[8,9]$.
Despite being a relatively common clinical entity, there is a paucity of literature regarding UAB. Additionally, we know relatively little about the natural history of UAB due to the lack of direct population-based studies [10]. Patients with poor bladder emptying due to $\mathrm{UAB}$ are potentially at risk of urologic complications, including recurrent UTI and bladder stones [1].

The disparity between UAB prevalence and the body of literature that encompasses our understanding of this condition persists despite a call for further research initiatives in an effort to better characterize UAB and develop future treatment options [7].

The aim of this study was to provide a review of our experience with UAB in a diverse patient population undergoing UDS for voiding dysfunction. We hope to better elucidate presenting symptoms, urodynamic findings, and possible risk factors for $\mathrm{UAB}$, while characterizing the epidemiology and treatment most often undertaken for this condition.

\section{MATERIALS AND METHODS}

After obtaining the approval of Institutional Ethics Review Board, we retrospectively reviewed the charts of consecutive patients undergoing multichannel video-urodynamic evaluation for voiding dysfunction by a single urologist between 2012 and 2014. Principles of the Helsinki declaration were followed. Patients were identified as fulfilling the criteria for DU, defined as a bladder contractility index (BCI) of less than 100, and the absence of identifiable $\mathrm{BOO}$ in patients who were able to void for pressure flow studies. This was calculated using the formula: $\mathrm{BCI}=\mathrm{PdetQmax}$ (detrusor pressure at peak flow rate) $+5 \mathrm{Qmax}$. Patients were grouped into those who were able to void, and those unable to void upon UDS. Charts were evaluated for patient demographics, presenting symptoms from a standard list of questions, urodynamic find-

Table 1. Demographics of patients evaluated for voiding dysfunction

\begin{tabular}{lc}
\hline Demographic & Value \\
\hline Total urodynamics evaluated & 343 \\
Patients meeting UAB criteria & $79 / 343(23.0)$ \\
Female sex & $54 / 79(68.4)$ \\
Follow-up (mo) & $11.1(0-34)$ \\
Age of UAB patients (yr) & $59.2(19-90)$ \\
\hline
\end{tabular}

Values are presented as number (\%) or mean (range). $\mathrm{UAB}$, underactive bladder. 
ings, suspected risk factors, and any short-term treatment. Descriptive statistics were utilized to present these findings.

\section{RESULTS}

During the study period, 343 consecutive patients underwent video-urodynamic evaluation. A total of 79 of 343 patients (23.0\%) were identified as meeting the criteria for DU. Demographics of the UAB cohort of patients are shown in Table 1.

The most common presenting symptoms in those identified with DU were urgency (63.3\%), weak stream (61.0\%), straining to void (57.0\%), and nocturia (48.1\%). A complete summary of symptoms of UAB patients is shown in Fig 1.

$\mathrm{UAB}$ patients were divided into those who were able to void for pressure flow studies (70.9\%) and nonvoiders/those with complete urinary retention (29.1\%) on UDS. UDS parameters are shown in Tables 2 and 3.

Of the 25 male UAB patients identified, $28.0 \%$ had undergone transurethral resection of the prostate, while $0.4 \%$ had undergone radical prostatectomy. The most prevalent UAB risk factors evaluated in this study population were prior pelvic surgery $(40.5 \%)$, medications promoting urinary retention (32.9\%), prior back surgery $(19.0 \%)$, and diabetes mellitus (11.4\%). A total of 10 of 79 patients (12.7\%) were treated with anticholinergic medication prior to evaluation.

Treatments undertaken were evaluated among those with $\mathrm{UAB}$ in the study cohort. Intermittent sterile catheterization alone was the predominant treatment modality in this group (54.4\%). Treatment modality breakdown is summarized in Fig. 2.

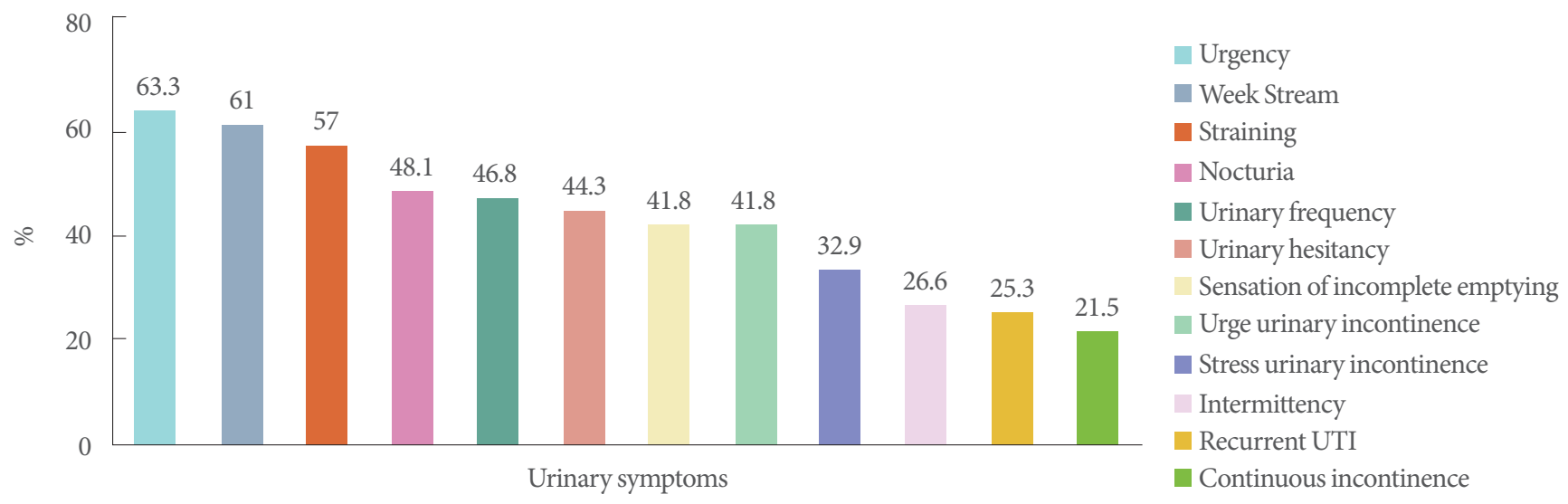

Fig. 1. Bar graph demonstrating prevalence of urinary symptoms among identified underactive bladder patients. UTI, urinary tract infection.

Table 2. Urodynamic parameters among "voiders" in the UAB patient cohort

\begin{tabular}{lc}
\hline Parameter & Value \\
\hline PdetQmax $\left(\mathrm{cm} \mathrm{H}_{2} \mathrm{O}\right)$ & $22.3(5-37)$ \\
Bladder contractility index & $67.5(20-94)$ \\
Qmax $(\mathrm{mL} / \mathrm{sec})$ & $9.3(1-22)$ \\
PVR of total volume $(\%)$ & $42.1(0-94)$ \\
Patients with concomitant detrusor & $19 / 79(24.1)$ \\
hyperactivity (DHIC) & \\
\hline
\end{tabular}

Values are presented as mean (range) or number (\%).

PdetQmax, detrusor pressure at peak flow rate; UAB, underactive bladder; Qmax, maximal flow rate; PVR, postvoid residual; DHIC, detrusor hyperactivity impaired contractility.
Table 3. Urodynamic findings in UAB patient cohort

\begin{tabular}{lc}
\hline Urodynamic finding & No. of patients (\%) \\
\hline $\begin{array}{l}\text { Concomitant detrusor } \\
\text { hyperactivity (DHIC) }\end{array}$ & $19 / 79(24.1)$ \\
Demonstrated SUI on UDS & $26 / 79(32.9)$ \\
Bladder diverticula on & $11 / 79(13.9)$ \\
fluoroscopy/cystoscopy & \\
SUI on history who demonstrated & $17 / 26(65.4)$ \\
SUI during UDS & \\
UUI on history who demonstrated & $11 / 33(33.3)$ \\
OAB on UDS &
\end{tabular}

UAB, underactive bladder; DHIC, detrusor hyperactivity impaired contractility; UDS, urodynamics; SUI, stress urinary incontinence; UUI, urge urinary incontinence; $\mathrm{OAB}$, overactive bladder. 


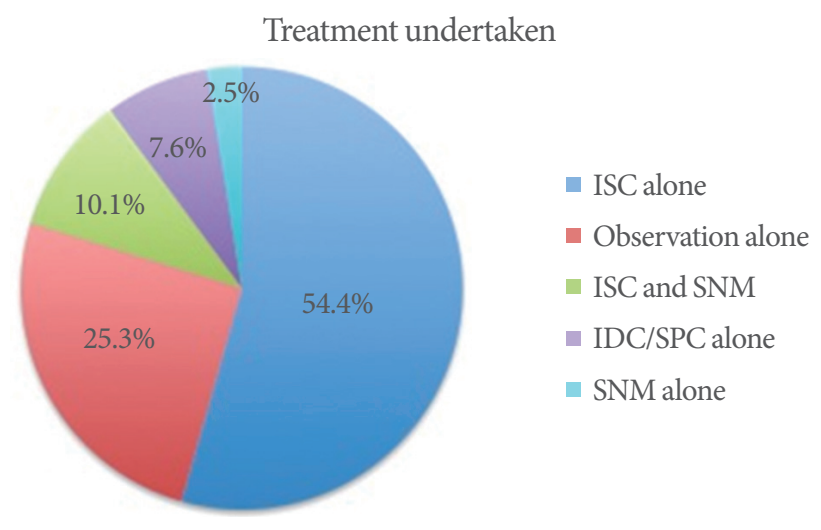

Fig. 2. Pie chart demonstrating treatment undertaken by underactive bladder patients in study cohort. ISC, intermittent sterile catheterization; SNM, sacral neuromodulation; IDC, indwelling catheter; SPC, suprapubic catheter.

\section{DISCUSSION}

$\mathrm{UAB}$ is a common cause of bladder dysfunction, and it has been reported to occur in approximately $9 \%-48 \%$ of men and $12 \%-45 \%$ of women presenting with nonneurogenic LUTS [11]. This was in keeping with the $23 \%$ patients identified with DU and other symptoms suggestive of UAB in our cohort of patients presenting with LUTS. A UDS study of incontinent institutionalized elderly patients demonstrated that the cause of incontinence was DU in $8 \%$, while over $30 \%$ had concomitant detrusor overactivity (DO) and DU, or detrusor hyperactivity impaired contractility (DHIC) as the cause of incontinence [12]. This is reflected in our study population where $24.1 \%$ of the patients had DHIC. This can be a difficult condition to treat for clinicians, as treating the DO component will invariably exacerbate the UAB component of their bladder dysfunction. Conversely, 22 of 33 patients, who reported urge urinary incontinence in history, failed to show evidence of OAB upon UDS. This highlights a potential pitfall in clinical practice. Many UAB patients may be misdiagnosed and receive empiric treatment for $\mathrm{OAB}$, as was the case for $12.7 \%$ of the patients in this cohort, potentially worsening their situation.

To best describe UABS, Osman et al. [11] have suggested a potential symptom-based definition. They proposed "reduced sensation of the need to void (the opposite of urgency) that may be associated with frequency and nocturia, or reduced voiding frequency often with the feeling of incomplete bladder emptying and incontinence that may predominate at nighttime". Interestingly in our cohort, the most common symptom cited by patients was urgency (63.3\%). This was followed by the more expected symptoms of weak stream, straining to void, and nocturia. UDS appears important in establishing a diagnosis of $\mathrm{UAB}$ as evidenced by the variable symptom profile with overlap between other common bladder disorders (OAB, BOO), in addition to the significant fraction of patients with DHIC. Due to this, an appropriate symptom-based definition will prove challenging to describe.

The high proportion of patients with UAB after prior pelvic surgery (40.5\%) and back surgery (19\%) raises an interesting point. It indicates that these neurogenic causes are significant risk factors for eventually developing $\mathrm{UAB}$, and the clinician should keep this in mind when assessing LUTS in those with prior back or pelvic surgery. The fact that $32.9 \%$ of patients were concurrently taking medications known to promote urinary retention should also act as a caution against prescribing these medications prior to detailed assessment or urodynamic evaluation of patients presenting with LUTS.

Based on the treatment undertaken in this study, it remains apparent that catheterization (intermittent and permanent) is a mainstay of bladder management ( $62 \%$ of the cases). There also appears to be a group where observation/conservative management may be appropriate, such as in those with some preservation of detrusor contractility, and without significant complications. In this study, a small subset of patients (2.5\%) was successfully treated with SNM alone, which has proven to be a promising treatment modality in appropriately selected patients [7].

Pharmacologic compounds, such as parasympathomimetics, have also been used to help bladder emptying in the management of UAB. While this certainly represents a promising avenue for future exploration, there has been minimal evidence to date demonstrating their clinical efficacy [13].

There are some limitations to this study that need to be noted. The study is limited by its retrospective nature. We expect that this limitation may be partially mitigated by collecting data from a single clinician. Moreover, the patient sample size was relatively small, hence it is difficult to predict if the data can be generalizable. It is clear that prospective studies will be extremely beneficial in collecting relevant data to assist in our understanding of UAB. There is also a lack of consensus on the optimal method to record bladder function. In this study, we used BCI to identify patients who met the criteria for UAB. A cutoff point of 100 was chosen to define DU for both men and women. Though a widely used formula, it may not represent the ideal measure of detrusor contractility due to the complexi- 
ty of UAB. It has been stated that even though BCI involves a simple calculation to identify patients with DU, it fails to adequately consider the coexistence of $\mathrm{BOO}$ [11].

In conclusion, UAB is a complex, but commonly encountered condition presenting with significant LUTS. There is significant overlap between symptoms of UAB and those of other bladder disorders, and the current burden of UAB is likely underestimated. UDS is the most significant measure for the accurate diagnosis of UAB. We conclude that there is a substantial need for further research in order to better characterize $U A B$ and understand its natural history.

\section{REFERENCES}

1. Chung E. Underactive bladder and detrusor underactivity: a review of pathophysiology and management strategies for this poorly understood bladder syndrome. Curr Bladder Dysfunct Rep 2014;9: 250-3.

2. Abrams P, Cardozo L, Fall M, Griffiths D, Rosier P, Ulmsten U, et al. The standardisation of terminology of lower urinary tract function: report from the Standardisation Sub-committee of the International Continence Society. Neurourol Urodyn 2002;21:167-78.

3. Chapple CR, Osman NI, Birder L, van Koeveringe GA, Oelke M, Nitti VW, et al. The underactive bladder: a new clinical concept? Eur Urol 2015;68:351-3.

4. Miyazato M, Yoshimura N, Chancellor MB. The other bladder syndrome: underactive bladder. Rev Urol 2013;15:11-22.

5. Jeong SJ, Kim HJ, Lee YJ, Lee JK, Lee BK, Choo YM, et al. Preva- lence and clinical features of detrusor underactivity among elderly with lower urinary tract symptoms: a comparison between men and women. Korean J Urol 2012;53:342-8.

6. Andersson KE. Bladder underactivity. Eur Urol 2014;65:399-401.

7. van Koeveringe GA, Vahabi B, Andersson KE, Kirschner-Herrmans R, Oelke M. Detrusor underactivity: a plea for new approaches to a common bladder dysfunction. Neurourol Urodyn 2011;30:723-8.

8. Peeters K, Sahai A, De Ridder D, Van Der Aa F. Long-term followup of sacral neuromodulation for lower urinary tract dysfunction. BJU Int 2014;113:789-94.

9. Al-zahrani AA, Elzayat EA, Gajewski JB. Long-term outcome and surgical interventions after sacral neuromodulation implant for lower urinary tract symptoms: 14 -year experience at 1 center. J Urol 2011;185:981-6.

10. Taylor JA 3rd, Kuchel GA. Detrusor underactivity: clinical features and pathogenesis of an underdiagnosed geriatric condition. J Am Geriatr Soc 2006;54:1920-32.

11. Osman NI, Chapple CR, Abrams P, Dmochowski R, Haab F, Nitti V, et al. Detrusor underactivity and the underactive bladder: a new clinical entity? A review of current terminology, definitions, epidemiology, aetiology, and diagnosis. Eur Urol 2014;65:389-98.

12. Resnick NM, Yalla SV, Laurino E. The pathophysiology of urinary incontinence among institutionalized elderly persons. N Engl J Med 1989;320:1-7.

13. Barendrecht MM, Oelke M, Laguna MP, Michel MC. Is the use of parasympathomimetics for treating an underactive urinary bladder evidence-based? BJU Int 2007;99:749-52. 